\title{
Microwave Plasmonics: A Novel Platform for RF Photonics
}

\section{Conference Paper}

Author(s):

Burla, Maurizio (D); Bonjour, Romain (D); Salamin, Yannick; Haffner, Christian (D); Heni, Wolfgang (D); Hoessbacher, Claudia; Fedoryshyn, Yuriy; Abrecht, Felix C.; Johnston, Peter V.; Elder, Delwin L.; Dalton, Larry R.; Leuthold, Juerg (iD)

Publication date:

2016

Permanent link:

https://doi.org/10.3929/ethz-b-000126497

Rights / license:

In Copyright - Non-Commercial Use Permitted 


\title{
Microwave Plasmonics: A Novel Platform for RF Photonics
}

\author{
Maurizio Burla ${ }^{1, *}$, Romain Bonjour ${ }^{1}$, Yannick Salamin ${ }^{1}$, Christian Haffner ${ }^{1}$, Wolfgang Heni ${ }^{1}$, Claudia Hoessbacher ${ }^{1}$, \\ Yuriy Fedoryshyn ${ }^{1}$, Felix C. Abrecht ${ }^{1}$, Peter V. Johnston ${ }^{2}$, Delwin L. Elder ${ }^{2}$, Larry R. Dalton ${ }^{2}$, and Juerg Leuthold ${ }^{1}$ \\ ${ }^{1}$ Institute of Electromagnetic Fields (IEF), ETH Zurich, Gloriastrasse 35, 8092 Zürich, Switzerland \\ ${ }^{2}$ Department of Chemistry, University of Washington, Seattle, WA 98195-1700, United States \\ *maurizio.burla@ief.ee.ethz.ch
}

(Invited)

\begin{abstract}
Plasmonic organic hybrid modulators recently demonstrated ultrafast operation $(>100 \mathrm{GHz})$, with ultra-compact footprints $\left(10 \mathrm{~s} \mu \mathrm{m}^{2}\right)$ and ultra-low power consumption (10s $\mathrm{fJ} / \mathrm{bit})$. We discuss the potential of the plasmonic platform for microwave photonics applications, with emphasis on mm-wave and sub- $\mathbf{T H z}$ wireless communications and signal processing.
\end{abstract}

Keywords-Plasmonics; integrated optics; wireless communications; microwave photonics.

\section{INTRODUCTION}

Next-generation $5 \mathrm{G}$ wireless communications promise to deliver 1,000 times more data traffic than current networks [1]. Radically novel approaches will be needed to support the required multi-Gbit/s or even Tbit/s data rates, including access to $\mathrm{mm}$-wave spectrum, massive MIMO techniques, and antenna beamsteering [2]. Microwave Photonics (MWP) allows to improve the performance of purely-RF systems by leveraging the broadband characteristics and flexibility of operation of photonics [3], and has been identified as an enabling technology for 5G [4]. In detail, MWP can assist with the aforementioned challenges e.g. by providing techniques for optical signal generation and distribution of $\mathrm{mm}$-waves towards antenna terminals or remote radio heads (RRH), optical control of phased arrays [4, 5], and more. However, many of those demonstrations have been performed employing expensive off-the-shelf discrete components. In large-scale applications, such as wireless communications, there is an absolute need to reduce size, weight, power and cost of the proposed MWP solutions. To achieve this goal, in recent years MWP systems have undergone a transformation towards on-chip photonic integration [6-8]. The aim of this effort is to enable the realization of integrated microwave photonic (IMWP) systems with lower bulk, power consumption and cost compared to the use of discrete components, while simultaneously retaining (or improving) performance and stability. As an additional challenge, $5 \mathrm{G}$ applications pose very stringent requirements on the speed of IMWP circuits. In fact, systems may need to operate at mm-wave or even sub-THz frequencies, in order to access the multi$\mathrm{GHz}$ bandwidths required for high data rates $[9,10]$. Comparably fast modulators (operating up to sub- $\mathrm{THz}$ speeds) with small power and footprint, fabricated using a low-cost, CMOS compatible process are not yet broadly available. Plasmonic modulators recently demonstrated ultra-fast operation $(>100 \mathrm{GHz})$, with ultra-compact footprints $\left(10 \mathrm{~s} \mu \mathrm{m}^{2}\right)$ and ultra-low power consumption (10s fJ/bit) [11-18]. The application of this novel platform to analog signal processing, and in particular to IMWP systems [7], is still largely to be explored, offering new frontiers and possibilities that could define the new field of Microwave Plasmonics.

Starting from a report on recent advances on plasmonics organic hybrid $(\mathrm{POH})$ devices, including plasmonic phase modulators [17], Mach-Zehnder [15, 18] and IQ-modulators [16], in this paper we discuss how those components can also be employed for ultrafast analog signal processing of microwave and mm-wave signals, with promising benefits. For these applications, in fact, POHs appear to be particularly attractive, mainly thanks to their high compactness and practically unlimited RF bandwidth [11].

\section{PlaSMONIC MODUlatorS}

\section{A. Principle of operation}

Plasmonic modulators are based on the concept of surface plasmon photonics, which deals with the generation, guiding and detection of surface plasmon polaritons (SPPs). SPPs are electromagnetic surface waves propagating at dielectric-metal interfaces $[11,17$, 19]. Melikyan et al. reported an ultra-compact plasmonic
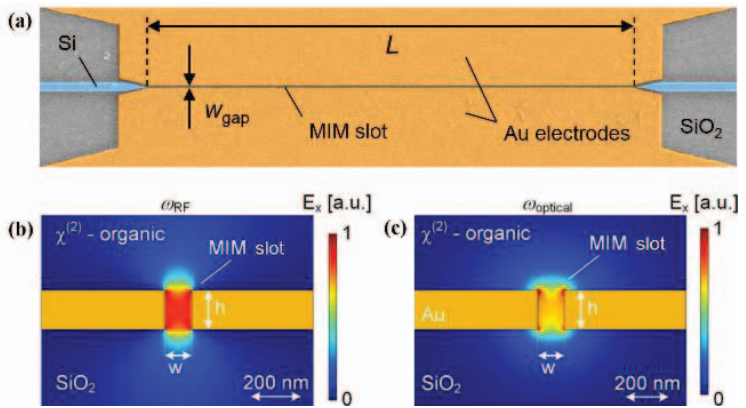

Fig. 1. SEM image (a) and magnitude of the RF (b) and optical (c) $E_{x}$ field component of a plasmonic phase modulator (from [17]) 
phase modulator only $29 \mu \mathrm{m}$-long and capable of highspeed operation over a bandwidth spanning more than $65 \mathrm{GHz}$ [17]. A typical plasmonic phase modulator [20], Fig. 1(a), consists in two gold electrodes forming a metalinsulator-metal (MIM) slot waveguide. The slot is filled with a nonlinear organic material $[21,22]$ whose refractive index change $\Delta n$ is linear with the electric field applied to the electrodes [17], according to Pockels effect [23]:

$$
\Delta n=\frac{1}{2} r_{33} n^{3} U / w_{\text {slot }}
$$

where $U$ is the modulating voltage, $w_{\text {slot }}$ is the gap width $r_{33}$ is the electro-optic coefficient of the nonlinear material. Light is fed to the plasmonic slot by means of a silicon strip waveguide. A linear taper transforms the photonic mode in the silicon nanowire to a SPP that propagates along the slot, where its phase is modulated by the applied field, and is then coupled back to the photonic waveguide mode by an identical output taper. High modulation indexes have been obtained thanks to the strong electrical and optical field confinement in the slot, and the excellent overlap of the optical and electrical field modes, as shown in Fig. 1(b,c) [17]. Thanks to the high modulation efficiency, the plasmonics slot can be kept short and, in turn, optical losses are reduced. A very important advantage of this kind of modulators for MWP applications is their practically unlimited RF bandwidth [18], due to the quasi-instantaneous nonlinear effect and the extremely small RC time constant of the structure. In fact, the small slot height and length reduce the capacitance to the femtofarad range, while the resistance is kept low thanks to the high conductivity of the metal electrodes. This yields a theoretical cutoff frequency in the $\mathrm{THz}$ range. Operation up to $110 \mathrm{GHz}$ was recently demonstrated [20], only limited by the available measurement instrumentation. This, in turn, enables operation in the mm-wave and sub-THz range desired in MWP applications. Another crucial advantage is compactness, which allows creating complex signal processing platforms on a chip [24, 25] with low footprint and cost.

\section{B. Plasmonic-photonic Mach-Zehnder Modulators}

$\mathrm{POH}$ phase modulators can be employed for intensity modulation by arranging them in a photonic MachZehnder interferometer structure, as shown by Heni et al. [19]. Fig. 2 shows an optical microscope image of an array of photonic interferometer structures where PPMs have been inserted in the two arms (inset) [26, 27]. The modulators are driven in a push-pull configuration by means of a ground-signal-ground RF probe. Hoessbacher et al. have experimentally demonstrated high speed optical interconnects up to $4 \times 36 \mathrm{Gbit} / \mathrm{s}$, based on dense plasmonic MZM arrays of such devices, where the modulators footprint is only limited by the size of the electrical contacts [27].

\section{All-plasmonic MZMs and IQ modulators}

An important step towards further miniaturization has been shown by integrating the Mach-Zehnder

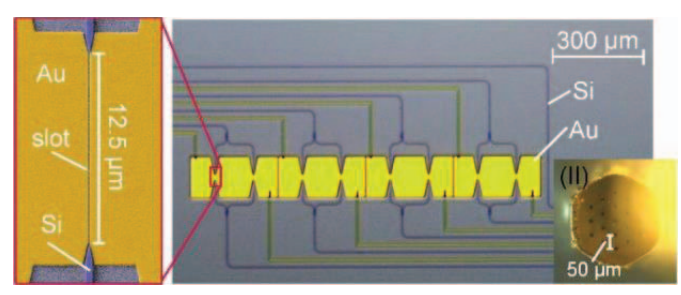

Fig. 2. Photonic-plasmonic MZM array from [27]

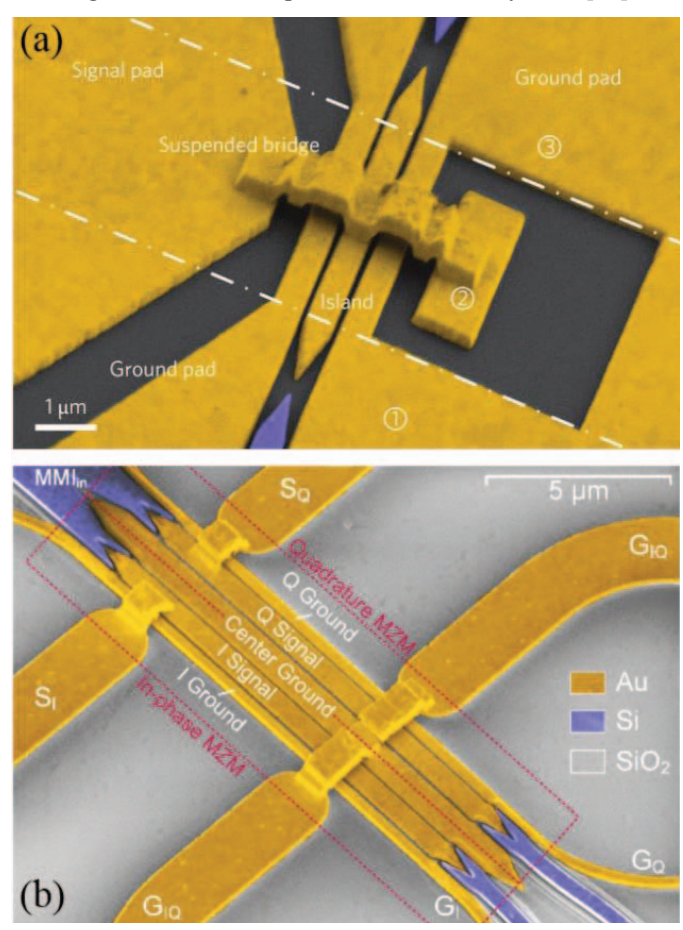

Fig. 3. False color SEM image of the plasmonic MZM from [18] (a) and the plasmonic IQ modulator from [16] (b)

interferometer in a fully-plasmonic structure. Haffner et al. demonstrated a plasmonic MZM with a record-small length of $10 \mu \mathrm{m}$ and losses as low as $8.1 \mathrm{~dB}$ that was successfully used in a data transmission experiment up to 72 Gbaud. Fig. 3a shows the structure [18]. A metallic island operates as central electrode of the MZM and is connected through a suspended bridge to the signal pad.

Recently, an all-plasmonic IQ modulator was presented in [16], featuring a footprint of $10 \times 75 \mu \mathrm{m}^{2}$, Fig. 3b. Besides being the basis for the implementation of complex modulation format in long-haul optical communications, IQ modulators are largely used in analog signal processing applications for arbitrary control over the modulation sidebands [28]. Therefore, this result could also be used to implement ultra-compact IQ modulators for on chip optical single-sideband (OSSB) generation, which is at the basis of many implementations of MWP signal processing functions such as optical true time delay lines, phase shifters, and instantaneous frequency measurement systems [7]. 


\section{NEW FRONTIERS FOR 5G ENABLED BY PLASMONICS}

\section{A. Co-integration of PPMs and antennas}

Recently, several research groups have addressed the challenge of direct conversion of free-space wireless radio signals to the optical domain, without resorting to electronic receivers, which become complex and expensive at sub-THz frequencies. Attempts have been made e.g. using lithium niobate modulators directly integrated with antennas, which showed the capability to convert signals up to $58 \mathrm{GHz}$ [29], or employing photonic crystal modulators on silicon coupled to bow-tie antennas [30]. However, these solutions either are limited by the speed of the modulators or display relatively large footprints, in the order of several $\mathrm{mm}^{2}$, precluding dense integration. Very recently, Salamin et al. demonstrated a promising solution to overcome those drawbacks by cointegrating a plasmonic phase modulator with a resonant antenna [31]. In this solution, the antenna arms are directly forming the plasmonics slot of the PPM (Fig. 4). The obtained "plasmotenna" achieves high efficiency thanks to several factors. In addition to the inherent advantages of the PPMs, designing the antenna arms to be resonant at the target RF frequency further enhances the voltage drop across the MIM slot. This demonstration has been performed at $60 \mathrm{GHz}$ [31], featuring a field enhancement in in the order of 35,000 , which allows to effectively convert incident electric fields in the order of $10 \mathrm{~V} / \mathrm{m}$. Further antenna configurations have recently been shown to improve even further the plasmotenna conversion efficiency [32]. Thanks to the broadband characteristics of the modulator, this scheme can also be extended to sub$\mathrm{THz}$ frequencies by properly scaling the size of the antenna. This should open the path towards fully transparent wireless links, not limited by the speed bottleneck of RF electronics, e.g. for last-mile network access to remote areas [10] or broadband wireless links to recover interruptions of fiber connections.

\section{B. Plasmonic beamforming networks}

In future $5 \mathrm{G}$ wireless communications, an important aspect will be the use of mm-wave frequency to access the needed bandwidth resources. Millimeter-waves are subject to larger free space path losses compared to microwave frequencies, and high gain, directive antennas will likely be required in practical deployments [1]. For this reason, antenna beam steering techniques are considered to provide connectivity to moving users. Optically controlled antennas are being proposed for seamless integration with radio-over-fiber networks [5]. Recently, a novel time and space division multiplexing technique has been proposed by Bonjour et al. as a possible way to increase the $5 \mathrm{G}$ cell capacity without increasing the cost and complexity of remote antenna terminals. Such technique is called symbol-by-symbol beam steering [33] and it consists in encoding the data aimed at different users in consecutive time slots. The antenna beam is then switched at the same speed as the bit period, such that consecutive transmitted bits are sent to

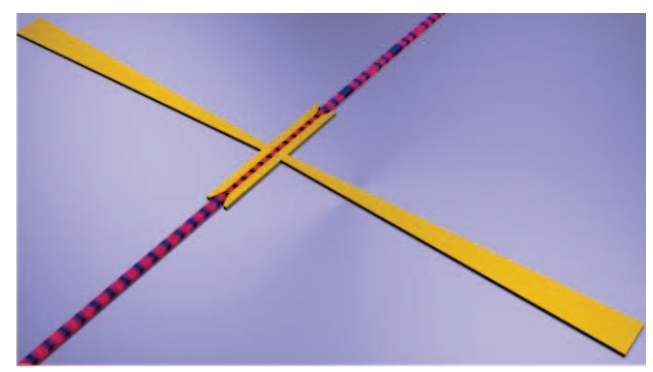

Fig. 4. Impression of the plasmotenna (from [31])

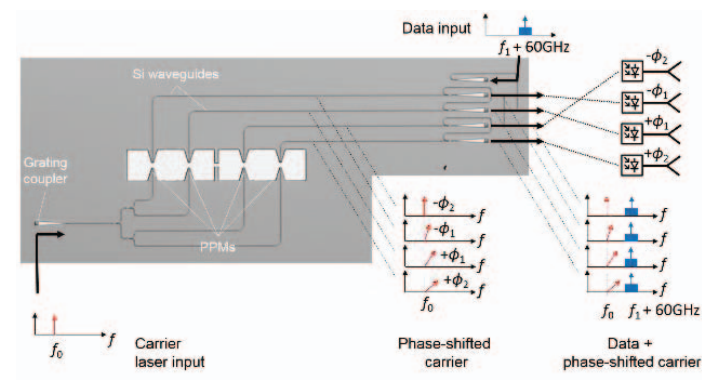

Fig. 5. Plasmonic beamformer based on PPMs [34]

different users. This technique requires beam switching speeds in the same order of magnitude as the bit duration. For high capacity multi-Gbit data links, this may reach a few picoseconds. Yet, most of current integrated beam steering systems are based on thermo-optical or electrooptical mechanisms that are limited to the microsecond or nanosecond speed range at best. Employing plasmonics phase modulators for beam control allows creating photonic beamforming systems with a steering speed only limited by the speed of the modulator itself, therefore able to reach tens of picosecond switching times or less. We recently fabricated a plasmonics beamformer for antenna arrays, consisting in an array of 4 PPMs used to control the electrical phase of the mm-wave signals radiated by the antennas of a linear antenna array. Fig. 5 shows an optical microscope picture of the plasmonic optical beamformer and illustrates the operating principle. A narrow-linewidth $\mathrm{CW}$ laser reference laser (frequency $f_{0}$ ) provides the optical carrier. Silicon grating couplers are used for fiberto-chip coupling of the laser light. A binary tree network of cascaded $1 \times 2$ MMI couplers is used to distribute the optical carrier to the 4 PPMs. Electrical signal are applied to the PPM electrodes via high-speed RF probes. The phase modulators operate by linear Pockels effect, as discussed in Sec. II.A. As a result, the instantaneous phase of the optical carrier at each modulator output experiences a phase shift that is proportional to the applied RF voltage. These phase-shifted carriers are then combined on-chip to another carrier centered at $f_{0}+60 \mathrm{GHz}$ and carrying the modulated data. The 4 outputs are fed into a photodetector array, where the RF signals to be transmitted by the antennas are generated via photonic mixing. Changing the relative phase shift induced by the PPMs ultimately induces equal changes the phase of the generated RF signal, thus allowing to control the beam propagation 
direction of the antenna array. Preliminary results of data transmission experiments show successful symbol-bysymbol steering capability up to 1 GBaud transmitted QPSK signals. Details of this demonstration will be reported in [33]. The data transmission speed of these preliminary results is being further optimized in current experiments and it is not limited by the plasmonic device. As a proof, we performed high-speed optical tests. Each modulator was driven by a $-3 \mathrm{dBm}$ RF signal while analyzing the optical modulation spectrum. The first order phase modulation sidebands show no frequency limitation up to $70 \mathrm{GHz}$ (constrained by the available signal generator only) with a ripple across the complete range of $\pm 1.5 \mathrm{~dB}$ and a maximum around $65 \mathrm{GHz}$.

\section{CONCLUSIONS}

We reported recent advances on plasmonics organic hybrid modulators and discussed their potential to realize novel IMWP functions. Demonstrations include direct wireless to optical conversion of mm-wave signals, and the creation of antenna feeding networks on a compact photonic chip, operating at $\sim 70 \mathrm{GHz}$ speeds. These results may enable novel techniques for $5 \mathrm{G}$ wireless applications, ultrafast mm-wave imaging, and more.

\section{ACKNOWLEDGMENTS}

The authors acknowledge the ERC PLASILOR project (670478), the National Science Foundation (DMR1303080) and the Air Force Office of Scientific Research (FA9550-15-1-0319) for partial funding of this work.

\section{REFERENCES}

[1] C. Dehos et al., "Millimeter-wave access and backhauling: the solution to the exponential data traffic increase in $5 \mathrm{G}$ mobile communications systems?," IEEE Comm. Mag., vol. 52, no. 9, pp. 88-95, 2014.

[2] F. Boccardi et al., "Five disruptive technology directions for $5 \mathrm{G}$," IEEE Comm. Mag., vol. 52, no. 2, pp. 74-80, 2014.

[3] J. Capmany, and D. Novak, "Microwave photonics combines two worlds," Nat. Photon., vol. 1, no. 6, pp. 319-330, 2007.

[4] R. Waterhouse, and D. Novak, "Realizing 5G: Microwave Photonics for 5G Mobile Wireless Systems," IEEE Microwave Magazine, vol. 16, no. 8, pp. 84-92, 2015.

[5] I. Feliciano da Costa et al., "Photonic Downconversion and Optically Controlled Reconfigurable Antennas in mm-waves Wireless Networks," OSA Technical Digest (online). p. W3K.3.

[6] S. Iezekiel et al., "RF Engineering Meets Optoelectronics: Progress in Integrated Microwave Photonics," IEEE Microw. Mag., vol. 16, no. 8 , pp. $28-45,2015$.

[7] D. Marpaung et al., "Integrated microwave photonics," Laser Photon. Rev., vol. 7, no. 4, pp. 506-538, 2013.

[8] M. Burla et al., "On-chip CMOS compatible reconfigurable optical delay line with separate carrier tuning for microwave photonic signal processing," Optics Express, vol. 19, no. 22, pp. 2147521484, 2011

[9] T. Nagatsuma et al., "Terahertz wireless communications based on photonics technologies," Opt. Express, vol. 21, no. 20, pp. $23736-$ 23747, 2013.

[10] S. Koenig et al., "Wireless sub-THz communication system with high data rate," Nat. Photon., vol. 7, no. 12, pp. 977-981, 2013.

[11] J. Leuthold et al., "Plasmonic Communications: Light on a Wire," Opt. Photonics News, vol. 24, no. 5, pp. 28-35, 2013.
[12] A. Emboras et al., "Electrically Controlled Plasmonic Switches and Modulators," IEEE J. Sel. Topics Quantum Electron., vol. 21, no. 4, pp. 276-283, 2015

[13] W. Cai et al., "Compact, High-Speed and Power-Efficient Electrooptic Plasmonic Modulators," Nano Letters, vol. 9, no. 12, pp. 4403-4411, 2009/12/09, 2009.

[14] J. S. Schildkraut, "Long-range surface plasmon electrooptic modulator," Appl. Optics, vol. 27, no. 21, pp. 4587-4590, 1988.

[15] W. Heni et al., "108 Gbit/s plasmonic Mach-Zehnder modulator with $>70 \mathrm{GHz}$ electrical bandwidth," J. Lightw. Technol., vol. 34, no. 2 , pp. 393-400, 2016.

[16] C. Haffner et al.,"Ultra-compact plasmonic IQ-modulator," in Proc. Europ. Conf. Opt. Comm. (ECOC 2015), pp. 1-3, 2015.

[17] A. Melikyan et al., "High-speed plasmonic phase modulators," Nat. Photon., vol. 8, no. 3, pp. 229-233, 2014.

[18] C. Haffner et al., "All-plasmonic Mach-Zehnder modulator enabling optical high-speed communication at the microscale," Nat. Photonics, vol. 9, no. 8, pp. 525, 2015.

[19] S. A. Maier, Plasmonics: Fundamentals and applications. Academic Press, 2007.

[20] C. Haffner et al., "Plasmonic Organic Hybrid Modulators - Scaling Highest Speed Photonics to the Microscale," Proc. IEEE, vol. PP, no. 99, pp. 1-18, 2016.

[21] W. Heni et al., "Optimizing Plasmonic Modulators for In-Device Nonlinearities of up to $275 \mathrm{pm} / \mathrm{V}$," in Advanced Photonics 2016 (IPR, NOMA, Sensors, Networks, SPPCom, SOF), OSA technical Digest (online) (Optical Society of America, 2016), p. ITu1A. 1.

[22] W. Jin, et al., "Structure-function relationship exploration for enhanced thermal stability and electro-optic activity in monolithic organic NLO chromophores," Journal of Materials Chemistry C, vol. 4, no. 15, pp. 3119-3124, 2016.

[23] R. W. Boyd, Nonlinear optics. Academic press, 2003.

[24] L. Zhuang et al., "Programmable photonic signal processor chip for radiofrequency applications," Optica, vol. 2, no. 10, pp. 854-859, 2015.

[25] J. Capmany, I. Gasulla, and D. Perez, "Microwave photonics: The programmable processor," Nat. Photon., vol. 10, no. 1, pp. 6-8, 2016.

[26] W. Heni et al., "High speed plasmonic modulator array enabling dense optical interconnect solutions," Optics Express, vol. 23, no. 23, pp. 29746-29757, 2015.

[27] C. Hoessbacher et al., "Optical Interconnect with Densely Integrated Plasmonic Modulator and Germanium Photodetector Arrays," OSA Tech. Digest. p. Th1F.6.

[28] I. Aryanfar et al., "Signal interference RF photonic bandstop filter," Optics Express, vol. 24, no. 13, pp. 14995-15004, 2016.

[29] Y. N. Wijayanto, H. Murata, and Y. Okamura, "60 GHz electrooptic modulator suspended to patch-antennas embedded with a gap on low-k dielectric material," 2013 Conference on Lasers and Electro-Optics Pacific Rim (CLEO-PR), Kyoto, 2013, pp. 1-2.

[30] X. Zhang et al., "Integrated Photonic Electromagnetic Field Sensor Based on Broadband Bowtie Antenna Coupled Silicon Organic Hybrid Modulator," J J. Lightw. Technol., vol. 32, no. 20, pp. 3774 3784, 2014.

[31] Y. Salamin et al., "Direct Conversion of Free Space Millimeter Waves to Optical Domain by Plasmonic Modulator Antenna," Nano Lett., vol. 15, no. 12, pp. 8342-8346, 2015.

[32] Y. Salamin et al., "Direct RF-to-Optical Detection by Plasmonic modulator integrated into a four-leaf-clover antenna," OSA Tech. Digest (2016). p. SM1E.6.

[33] R. Bonjour et al., "Ultra-Fast Millimeter Wave Beam Steering," IEEE J. Sel. Topics Quantum Electron., vol. 52, no. 1, pp. 1-8, 2016.

[34] R. Bonjour et al., "Plasmonic Phased Array Feeder enabling Symbol-by-Symbol mm-Wave Beam Steering at $60 \mathrm{GHz}$ " Proc. Int. Top. Meeting on Microwave Photonics 2016, unpublished. 